\title{
Coronary artery fistula and premature coronary atherosclerosis
}

\author{
Łukasz Turek ${ }^{1}$, Anna Polewczyk ${ }^{1,2}$, Marianna Janion ${ }^{1,2}$, Marcin Sadowski ${ }^{2,3}$ \\ ${ }^{1} 2^{\text {nd }}$ Department of Cardiology, Swietokrzyskie Cardiology Center, Kielce, Poland \\ ${ }^{2}$ Medical Faculty and Health Sciences, The Jan Kochanowski University, Kielce, Poland \\ ${ }^{3}$ Catheterization Laboratory, Swietokrzyskie Cardiology Center, Kielce, Poland
}

A 48-year-old man with insignificant family history and without cardiovascular risk factors was admitted due to typical retrosternal chest pain of 30 min duration. Physical examination was within normal limits. Transthoracic echocardiography revealed anterior wall basal segments hypokinesis. Signs of ongoing myocardial ischemia in admission electrocardiogram (ST-segment depression in $\mathrm{V}_{1}-\mathrm{V}_{4}$ ) together with a significant rise in cardiac troponin $\mathrm{T}$ level (from 4.9 to $143.4 \mathrm{ng} / \mathrm{L}$ ) resulted in a diagnosis of acute coronary syndrome without ST-segment elevation as most probable. Coronary angiography revealed a critical stenosis of a marginal branch (Fig. 1A) and coronary artery fistula (CAF) originating from the left main coronary artery (Fig. 1C, D). A successful percutaneous coronary intervention of the marginal branch with drug-eluting stent implantation was performed (Fig. 1B). The patient's further recovery was uneventful. A repeat careful echocardiographic examination was able to detect flow to the right pulmonary artery (Fig. 1E, F). Moreover, diameter measurements of cardiac chambers, pulmonary and systemic flow ratios $(\mathrm{Qp} /$ $/ \mathrm{Qs}$ ) and systolic pulmonary artery pressure determined by Doppler echocardiography were normal. Multidetector computed tomography is commonly used to detect and enhance visualization of the complex geometry of coronary fistulas, however, in this case the computed tomography scan was not performed related to an absence of pressure and volume overload on echocardiographic study, the patient was asymptomatic with CAF (unexplained relation of CAFs to incidence of atherosclerotic coronary artery disease) and radiological protection. This patient was recommended conservative management of $\mathrm{CAF}$ as the first-line treatment option and further follow-up. CAF is a rare vascular anomaly with an estimated prevalence of $0.002 \%$ in the general population and it can reach up to $5 \%$ in patients undergoing coronary angiography. In adults, about $30 \%$ of CAF cases are associated with coronary atherosclerosis - however, the relationship between CAFs and coronary atherosclerosis has not yet been clarified.

Conflict of interest: None declared

Address for correspondence: Łukasz Turek, $2^{\text {nd }}$ Department of Cardiology, Swietokrzyskie Cardiology Center, ul. Grunwaldzka 45, 25-736 Kielce, Poland, tel: +48 692199654, e-mail: garbit.garbit@interia.pl

Received: 24.11.2018 Accepted: 4.02.2019 


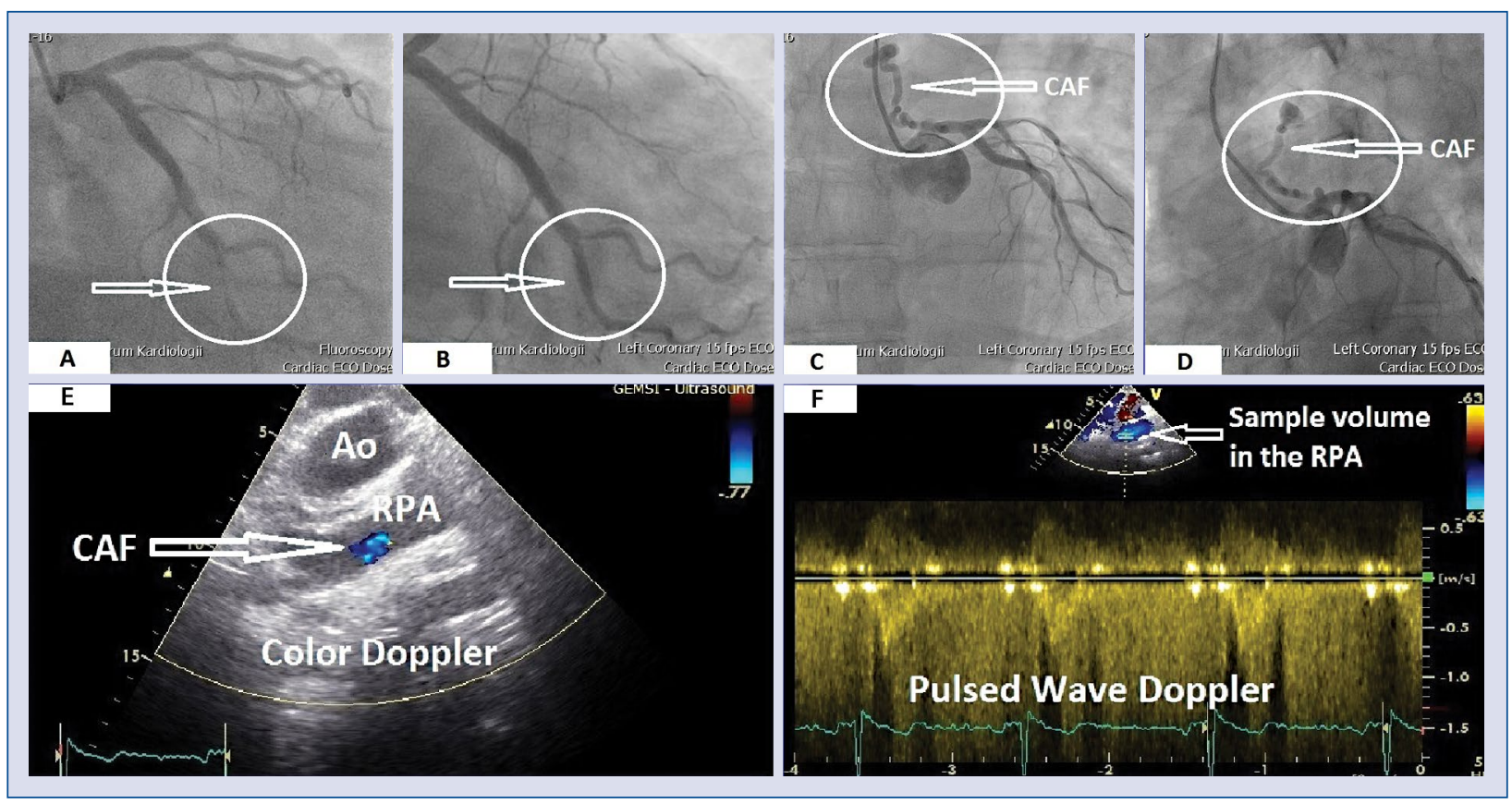

Figure 1. A. Coronary angiography: critical stenosis of the marginal branch (arrow); B. Coronary angiography: successful percutaneous coronary intervention of marginal branch (arrow); C, D. Coronary angiography: coronary artery fistula (CAF; arrow); E. Echocardiography study - suprasternal view of long axis of right pulmonary artery (RPA) - color flow Doppler images: CAF flow (arrow); F. Echocardiography study - suprasternal view of long axis of RPA — pulsed-wave Doppler: CAF flow (arrow) - sample volume in the RPA; Ao - aortic arch. 